\title{
Incisal margin condition after LEEP for cervical intraepithelial neoplasia patients and prognosis
}

\author{
HONG CHEN ${ }^{1}$, XIUFEANG LIU ${ }^{2}$ and LINA XU ${ }^{3}$ \\ Departments of ${ }^{1}$ Gynaecology and ${ }^{2}$ Science and Education, Binzhou City Center Hospital, Binzhou, \\ Shandong 251700; ${ }^{3}$ Department of Gynaecology, Jining No. 1 People's Hospital, Jining, Shandong 272111, P.R. China
}

Received March 17, 2016; Accepted May 11, 2016

DOI: $10.3892 /$ etm.2016.3338

\begin{abstract}
The aim of the study was to examine the relationship between incisal condition after loop electrosurgical excision procedure (LEEP) operation for cervical intraepithelial neoplasia (CIN) patients and prognosis. A study of high-risk incisal margin positive cases was also performed. We compared the differences in the prognosis of the 1-year follow-up visit in the $120 \mathrm{CIN}$ patients admitted to the hospital during the period from April 2013 to April 2014. A total of 43 cases of positive incisal margin (35.8\%), and 77 negative cases were included in the study. The differences in age, course of disease, and CIN level between the two groups showed no statistical significance $(\mathrm{P}>0.05)$. In the positive group, the positive ratio was significantly higher than that of the negative group $(\mathrm{P}<0.05)$. The positive incisal margin showed a significant positive correlation with human papillomavirus (HPV) positivity $(\mathrm{r}=0.327, \mathrm{P}=0.035)$. The condition of most patients with positive incisal margin and HPV was critical, which was followed by positive incisal margin and negative HPV. The patients with the best prognosis were those with significant negative incisal margin as well as HPV $(\mathrm{P}<0.05)$. In conclusion, the positive incisal margin after LEEP operation was associated with relapse of the disease. Thus, considering the HPV test into consideration is valuable for the prognosis of the disease.
\end{abstract}

\section{Introduction}

Loop electrosurgical excision procedure (LEEP) operation exploited tissue impedance for the generation of a large amount of heat in order to instantly for cleavage of the lesion (1). The operation was simple under the condition of local anesthesia, led to little trauma and few complications and the procedure was relatively inexpensive.

Correspondence to: Dr Hong Chen, Department of Gynaecology, Binzhou City Center Hospital, 108 South Huancheng Road, Binzhou, Shandong 251700, P.R. China

E-mail: chen_hong1122@163.com

Key words: cervical intraepithelial neoplasia, loop electrosurgical excision procedure, incisal margin condition, human papillomavirus
Currently, LEEP is widely applied in gynecological diseases including cervical disease, especially in cervical intraepithelial neoplasia (CIN) (1). The findings of a previous study showed that, positive incisal margin condition following LEEP operation was closely associated with the relapse or deterioration of the disease (2). High-risk factors include CIN level, age and human papillomavirus (HPV) infection (3).

In the present study, we examined the role of positive incisal margin condition following LEEP operation and HPV infection in the progression of the disease.

\section{Patients and methods}

Patients. A total of 120 CIN patients admitted to the Binzhou City Center Hospital during the period April 2013 to April 2014 were selected consecutively. The patients were diagnosed using multipoint uterine curettage and were deemed pathologically suitable for LEEP operation. Written informed consent was provided by the patients and their families. Approval for the study was provided by the ethics committee of the Binzhou City Center Hospital (Shandong, China).

Patients with previous experience of cervix operations, trauma, multiple LEEP operations, or failed LEEP operations were excluded. Those exhibiting other gynecological diseases such as cancer, fibroids, endometriosis and gynecologic inflammation, and those with autoimmune disease, chronic infection, diabetes mellitus, or vital organ complications were also excluded. The patients who failed to follow precautions, follow-up visits or had insufficient clinical data were also excluded. The patients were aged 34-74 years, with an average of $47.8 \pm 10.2$ years. There were 37 cases of CIN level I, 56 cases of CIN level II, and 27 cases of CIN level III. Pregnancy had occurred 1-3 times, with an average of 1.8 times, and giving birth was 1-3 times, with an average of $1.5 \pm 0.4$ times.

Methods. The process of LEEP operation included emptying the bladder first. Following disinfection of the operating table, the patients were placed in a lithotomy position and covered in drape. The speculum was used to expose the cervix uteri, Logo solution was smeared on the surface and the boundary of the nidus were shown. During the operation, a large size of electrode was used [Shenzhen Jinkewei Technology Co., Ltd. (Shenzhen, China), in the high-frequency electric radiosurgical knife with the frequency of $3.8 \mathrm{MHz}$, the thermal output power and electrocoagulation 
Table I. Comparison of the general data of patients with different incisal margin conditions.

\begin{tabular}{|c|c|c|c|c|c|}
\hline Incisal margin condition & Average age, years & Average disease course, years & CIN level I & CIN level II & CIN level III \\
\hline Positive, $n=43$ & $47.6 \pm 10.1$ & $2.6 \pm 0.7$ & $13(30.2 \%)$ & $22(51.2 \%)$ & $8(18.6 \%)$ \\
\hline Negative, $\mathrm{n}=77$ & $47.7 \pm 10.2$ & $2.8 \pm 0.6$ & $24(31.2 \%)$ & $34(44.2 \%)$ & $19(24.7 \%)$ \\
\hline t-test & 0.867 & 0.576 & & 0.750 & \\
\hline P-value & 0.523 & 0.314 & & 0.687 & \\
\hline
\end{tabular}

CIN, cervical intraepithelial neoplasia.

was 30-40 W] for the lesion resection including resection of the surrounding infiltrated tissue. If the lesion section was extremely large and surpassed the width of the electrode, the lesion was divided into sections and a small size square shape electrode or circle electrode was used. The electrode was moved slowly from left to right, or up and down to cut the tissue, at a depth of $1.0-2.5 \mathrm{~cm}$. Following the operation, the margins were marked to observe the incisal margin condition (negative or positive results of TCT for the incisal margin tissues). The sample from the operation was preserved in $10 \%$ formaldehyde solution prior to the pathological examination. The pathological results were assessed by two experts independently, and in case of disagreement consensus was reached via a third expert.

HPV screening method. A specific HPV sampler (Kaipu, Hong Kong, China) was inserted into the cervix uterus, rotated clockwise for 3 rounds and then stopped for $10 \mathrm{sec}$. Subsequently, the sampler was removed slowly and placed in preserving fluid. The samples were kept at room temperature prior to testing. An HPV-DNA extraction kit (Qiagen, Hilden, Germany), HPV gene microarray parting test kit (Kaipu), PE9600 PCR amplifier (Applied Biosystems, Foster City, CA, USA), and HybriMax medical nucleic acid fast hybridization device (Kaipu) were used. The tests were carried out and analyzed by the experts.

Procedure for follow-up visit. After 1-year follow-up visit, the HPV and TCT tests were carried out regularly. In case of any abnormal symptoms, colposcopy was employed. Attention was given to the incisal margin condition of the patients after the operation and then divided into positive and negative groups. The age, disease course, CIN level and HPV positive ratio between the groups were compared to develop a correlation between the positive incisal margin and positive HPV.

Statistical analysis. SPSS 19.0 software (SPSS, Inc., Chicago, IL, USA) was employed to input and analyze the data. Data were presented as mean \pm standard deviation. Comparisons between the groups were made using a t-test. Enumeration data were expressed as a ratio or percentage. The $\chi^{2}$ test was applied for comparison among the groups. In terms of the correlative analysis for the qualitative data, the $\chi^{2}$ test was used. $\mathrm{P}<0.05$ was considered to indicate a statistically significant difference.

\section{Results}

Analysis of incisal margin condition of patients. Following pathological diagnosis of the 120 cases included in the present
Table II. Comparison of the human papillomavirus rate among patients with different incisal margin conditions [cases (\%)].

\begin{tabular}{lccccc}
\hline & \multicolumn{2}{c}{$\begin{array}{c}\text { Follow-up visits } \\
\text { for } 6 \text { months }\end{array}$} & & \multicolumn{2}{c}{$\begin{array}{c}\text { Follow-up visits } \\
\text { for } 12 \text { months }\end{array}$} \\
\cline { 2 - 3 } Incisal margin & Positive & Negative & & Positive & Negative \\
\hline Positive, $\mathrm{n}=43$ & $21(48.8)$ & $22(51.2)$ & & $29(67.4)$ & $14(32.6)$ \\
Negative, $\mathrm{n}=77$ & $23(29.9)$ & $54(70.1)$ & & $19(24.7)$ & $58(75.3)$ \\
$\chi^{2}$-test & 4.274 & & 21.027 & \\
P-value & 0.039 & & $<0.001$ & \\
\hline
\end{tabular}

study, 43 cases $(35.8 \%)$ showed positive incisal margin, and 77 cases showed negative incisal margin (Table I). The differences in age, disease course and CIN level of the positive and negative groups showed no statistical significance $(\mathrm{P}>0.05)$.

Comparison of the HPV-positive rate in patients with different incisal margin conditions. In the follow-up visits after 6 and 12 months, the HPV-positive rate of the positive incisal margin group was significantly higher than that of the negative group $(\mathrm{P}<0.05)$ (Table II). The related analysis revealed a positive correlation between the positive incisal margin and positive HPV ( $r=0.327, \mathrm{P}=0.035)$.

Relationship between incisal margin condition, positive HPV and the prognosis of disease. The disease deterioration rate in the patients with positive incisal margin and positive HPV was the highest at $79.3 \%$ (23/29). It was followed by the patients with a positive incisal margin and negative HPV, which accounted for 50.0\% (7/14). Those with negative incisal margin and positive HPV accounted for 47.4\% (9/19). Furthermore, the patients with a negative incisal margin and negative HPV exhibited best prognosis, with the disease deterioration rate of $10.3(6 / 58)$. The comparison among the groups showed statistical significance $\left(\chi^{2}=41.601, \mathrm{P}<0.001\right)$.

\section{Discussion}

Previous findings have shown that, following CIN treatment, the possibility for disease to develop into invasive cervical cancer is 10-fold higher than that of healthy individuals (4). Clinically, LEEP operation is often used for treatment. The lesion following operation potentially leads to different 
degrees of residue. The lesion would continue to exist and even develop into invasive cancer. Therefore, the incisal margin condition following the operation has attracted much attention in the clinic (5). It has been shown that, the positive rate for the incisal margin of conization of cervix for CIN is $5-40 \%$, while in China it is $35.8 \%$ (6).

In the LEEP operation, the circled metal wire has been used to conduct high-frequency low-voltage electric waves. When contacting the lesion tissue, because of the impedance from the tissue itself, the electric wave instantly generates a large amount of heat to accomplish the electrotomy and electrocoagulation simultaneously (5). This operation has been applied in the treatment of early-stage cervical invasive cancer and CIN in recent years. The pathological incisal margin condition is the important index for CIN development (7). It has been proven in the clinic (8) that patients with a positive incisal margin have poor prognosis, and it is a high risky factor for potential residue and relapse of the disease. In the present study, we analyzed the general data of the patients with a positive and negative incisal margin. The results demonstrate that there was no statistical significance in the age, disease course and CIN level of the two groups. The post-operation positive incisal margin was not significantly associated with age, disease course or the CIN level of the patients. Patients of any age, disease course, and CIN level may exhibit a positive incisal margin after the operation (9). Attention should be paid to the patients who have undergone LEEP operation, and should be required to attend timely follow-up visits.

As the HPV test has become widely applied, medical staff gradually realized the effect of the HPV infection after the operation on the relapse and sustained development of CIN. At present, an HPV test carried out 6 months after the LEEP operation constitutes a predictive factor suggesting sensitivity for CIN relapse is as high as $90 \%$, which is significantly higher than the cytological $70 \%$ (10). The HPV test remains valid for two years subsequent to the operation. The study results of the group showed that the HPV-positive ratio of the patients with positive incisal margin was significantly higher than that of the negative group, and there was a positive correlation between positive incisal margin and HPV positive ratio. The HPV continuous infection rate of positive incisal margin may lead to further development (11) of the disease as the time period becomes longer due to incomplete treatment. Positive incisal margin is a high risk factor for causing positive HPV. Thus, the patients who had positive incisal margin and kept the cervix uteri should be monitored. The results obtained in the present study concur with those of previous studies (12).

The present study further analyzed the incisal margin, positive HPV and prognosis of the patients. The results have shown that the patients with positive incisal margin and HPV had the highest deterioration rate. The patients with a negative incisal margin and HPV exhibited the best prognosis.

In summary, the positive incisal margin following LEEP operation is linked to relapse of the disease. If combined with an HPV test, it is of some value for the prognosis of this disease. Therefore, after the operation, the high risk of the same sub-type HPV may indicate the future development of the CIN lesion. When performing the LEEP operation, the lesion tissue should be carefully distinguished and, if possible, be eliminated thoroughly, to avoid positive incisal margin, which would cause disease deterioration (13). If the disease deteriorates, it may develop into cervical invasive cancer, and would have adverse effects on the life of the patients (14). Therefore, the patients are to strictly comply with the schedule for the follow-up visits subsequent to the operation.

\section{References}

1. Santesso N, Mustafa RA, Wiercioch W, Kehar R, Gandhi S, Chen Y, Cheung A, Hopkins J, Khatib R, Ma B, et al: Systematic reviews and meta-analyses of benefits and harms of cryotherapy, LEEP, and cold knife conization to treat cervical intraepithelial neoplasia. Int J Gynaecol Obstet 132: 266-271, 2016.

2. Wong AS, Li WH and Cheung TH: Predictive factors for residual disease in hysterectomy specimens after conization in early-stage cervical cancer. Eur J Obstet Gynecol Reprod Biol 199: 21-26, 2016.

3. Fallala MS and Mash R: Cervical cancer screening: Safety, acceptability, and feasibility of a single-visit approach in Bulawayo, Zimbabwe. Afr J Prim Health Care Fam Med 7: 3-4, 2015.

4. Elit L, Levine MN, Julian JA, Sellors JW, Lytwyn A, Chong S, Mahony JB, Gu C, Finch T and Zeferino LC: Expectant management versus immediate treatment for low-grade cervical intraepithelial neoplasia: a randomized trial in Canada and Brazil. Cancer 117: 1438-1445, 2011

5. Song JS, Hwang I and Gong G: Outcome of 'atypical squamous cells' in cervical cytology: Follow-up assessment by loop electrical excision procedure. Korean J Pathol 46: 359-364, 2012.

6. Wu J, Jia Y, Luo M and Duan Z: Analysis of residual/recurrent disease and its risk factors after loop electrosurgical excision procedure for high-grade cervical intraepithelial neoplasia. Gynecol Obstet Invest: Sep 3, 2015 (Epub ahead of Print).

7. Liverani CA, Di Giuseppe J, Clemente N, Delli Carpini G, Monti E, Fanetti F, Bolis G and Ciavattini A: Length but not transverse diameter of the excision specimen for high-grade cervical intraepithelial neoplasia (CIN 2-3) is a predictor of pregnancy outcome. Eur J Cancer Prev: Aug 27, 2015 (Epub ahead of Print).

8. Ruano Y, Torrents M and Ferrer FJ: Human papillomavirus combined with cytology and margin status identifies patients at risk for recurrence after conization for high-grade cervical intraepithelial neoplasia. Eur J Gynaecol Oncol 36: 245-251, 2015.

9. Malapati R, Chaparala S and Cejtin HE: Factors influencing persistence or recurrence of cervical intraepithelial neoplasia after loop electrosurgical excision procedure. J Low Genit Tract Dis 15: 177-179, 2011.

10. Monsonego J, Pintos J, Semaille C, Beumont M, Dachez R Zerat L, Bianchi A and Franco E: Human papillomavirus testing improves the accuracy of colposcopy in detection of cervical intraepithelial neoplasia. Int J Gynecol Cancer 16: 591-598, 2006.

11. Alvarez RD, Huh WK, Bae S, Lamb LS Jr, Conner MG, Boyer J, Wang C, Hung CF, Sauter E, Paradis M, et al: A pilot study of pNGVL4a-CRT/E7(detox) for the treatment of patients with HPV16 cervical intraepithelial neoplasia 2/3 (CIN2/3). Gynecol Oncol 140: 245-252, 2016.

12. Kang WD, Ju UC and Kim SM: A human papillomavirus (HPV)-16 or HPV-18 genotype is a reliable predictor of residual disease in a subsequent hysterectomy following a loop electrosurgical excision procedure for cervical intraepithelial neoplasia 3. J Gynecol Oncol 27: e2, 2016.

13. Kang WD and Kim SM: Human papillomavirus genotyping as a reliable prognostic marker of recurrence after loop electrosurgical excision procedure for high-grade cervical intraepithelial neoplasia (CIN2-3) especially in postmenopausal women. Menopause 23: 81-86, 2016.

14. Mo LZ, Song HL, Wang JL, He Q, Qiu ZC and Li F: Pap smear combined with hpv testing: A reasonable tool for women with high-grade cervical intraepithelial neoplasia treated by LEEP. Asian Pac J Cancer Prev 16: 4297-4302, 2015. 